\title{
Shock agudo secundario a un neuroblastoma congénito. Caso clínico neonatal
}

\author{
Acute shock secondary to congenital neuroblastoma. Neonatal case report
}

\author{
Lic. Ignacio Mastro-Martínez $z^{a}$ Lic. María J. Pérez-Ortega ${ }^{a}$, Lic. Emilia Urrutia ${ }^{b}$, Lic. María J. Ortega-Acosta y \\ Lic. Francisco J. Pacheco Sánchez-lafuentec
}

\begin{abstract}
RESUMEN
El neuroblastoma congénito es el tumor sólido maligno más frecuente en el período neonatal. La forma de presentación suele ser por diagnóstico prenatal o por una masa abdominal. Su estadificación permite clasificarlo en grupos de riesgo con pronóstico y tratamiento diferentes. En el período neonatal, se caracteriza por la alta tasa de regresión espontánea y el buen pronóstico (supervivencia libre de enfermedad a los 5 años superior al $90 \%)$.

Se presenta un caso clínico de neuroblastoma congénito cuya forma de presentación, shock ehipertensión, solo estaba descrita en otra ocasión antes. El tratamiento antihipertensivo, junto con la quimioterapia sistémica, produjo el control clínico y la mejoría del paciente.

Palabras clave: neuroblastoma congénito, shock, hipertensión, neonatología.
\end{abstract}

\begin{abstract}
Congenital neuroblastoma is the most frequent malignant solid tumor in the neonatal period. The clinical presentation is usually either by prenatal diagnosis or by palpation of an abdominal mass. Staging allows classifying it according to risk groups with a different prognosis and treatment. In the neonatal period, it is characterized by a high rate of spontaneous regression and good prognosis (disease-free survival at 5 years greater than $90 \%$ ).

We present a clinical case of congenital neuroblastoma whose presentation, shock and hypertension, was only described on a previous occasion. Antihypertensive treatment along with systemic chemotherapy produced clinical control and patient improvement.
\end{abstract}

Key words: congenital neuroblastoma, shock, hypertension, neonatology.

http:/ / dx.doi.org/10.5546/ aap.2019.e163

a. Unidad de Gestión Clínica de Pediatría, Baza (España).

b. Hospital Universitario Virgen de las Nieves, Servicio de Pediatría y Oncohematología Pediátricas, Granada (España).

c. Hospital Universitario Virgen de las Nieves, Servicio de Pediatría, Unidad de Cuidados Intensivos Pediátricos, Granada (España).

Correspondencia:

Lic. Ignacio Mastro-Martínez:

ignacio.mastro.sspa@juntadeandalucia.es

Financiamiento: Ninguno.

Conflicto de intereses: Ninguno que declarar.

Recibido: 22-5-2018

Aceptado: 20-10-2018
Cómo citar: Mastro-Martínez I, Pérez-Ortega MJ, Urrutia E, Ortega-Acosta MJ, Pacheco Sánchez-lafuente FJ. Shock agudo secundario a un neuroblastoma congénito. Caso clínico neonatal. Arch Argent Pediatr 2019;117(2):e163-e166.

\section{INTRODUCCIÓN}

El neuroblastoma es el segundo tumor sólido más frecuente en la infancia, que supone el 7-10\% de los tumores infantiles (incidencia aproximada de 10 casos por millón de niños menores de 15 años). ${ }^{1}$ El neuroblastoma congénito es el tumor sólido más frecuente en el neonato (el 30-50 \% de los tumores malignos). ${ }^{2}$ El diagnóstico se suele realizar intraútero mediante ecografía o al nacer tras la palpación de una masa abdominal. Otro tipo de presentaciones, [(síndrome de Horner, compresión espinal y paraparesia de miembros inferiores, síndrome de opsoclono-mioclonos, o la afectación cutánea (blueberry muffin baby)] son menos frecuentes. ${ }^{3}$

El sistema de clasificación surge como consenso del International Neuroblastoma Risk Group (INRG), basado en factores de riesgo definidos por los criterios clínicos y las pruebas de imagen al momento del diagnóstico. ${ }^{4}$ Debido a las características únicas del neuroblastoma en el período neonatal, este grupo tiene su propio estadio, que se caracteriza por alta tasa de regresión espontánea y buen pronóstico (supervivencia libre de enfermedad superior al $90 \%$ ). ${ }^{1}$

Se presenta un caso clínico de neuroblastoma congénito cuya forma de presentación, shock e hipertensión, solo estaba descrita en otra ocasión.

\section{Descripción del caso}

Recién nacido con 1 hora de vida que ingresó por dificultad respiratoria y palidez intensa. Embarazo sin incidencias con ecografías normales, parto eutócico a las 39 semanas, líquido amniótico meconial, puntuación del test de Apgar 9/10, gasometría arterial de cordón normal, no precisó reanimación. A la hora de vida, mal estado general, taquipnea, tiraje subcostal, quejido audible sin fonendo, 
hipotonía generalizada y mala perfusión con palidez. Presentaba taquicardia e hipertensión (tensión arterial: 97/76 mmHg; frecuencia cardíaca -FC-: 180 latidos por minuto-lpm-). En la gasometría venosa, presentaba acidosis metabólica grave ( $\mathrm{pH}$ : 6,9; presión parcial de dióxido de carbono: $50 \mathrm{mmHg}$; bicarbonato: $9 \mathrm{mmol} / \mathrm{L}$; exceso de base: $-18,7 \mathrm{mmol} / \mathrm{L}$; láctico: $10,7 \mathrm{mmol} / \mathrm{L}$ ), con hemograma y bioquímica normales, que incluían reactantes de fase aguda (proteína $\mathrm{C}$ reactiva: $0 \mathrm{mg} / \mathrm{dl}$; procalcitonina: $0,79 \mathrm{ng} / \mathrm{ml}$ ).

Durante la estabilización, persistía la hipertensión con tensión arterial media superior a $80 \mathrm{mmHg}$. Se intubó y se administró bicarbonato en dos ocasiones, con persistencia de acidosis grave e hiperlactacidemia, hipertensión y elevación moderada de transaminasas y plaquetopenia. Aunque no presentaba factores de riesgo infeccioso y los reactantes de fase aguda eran normales, se administró ampicilina y gentamicina intravenosa. Se realizó un ecocardiograma transtorácico que mostró función cardíaca normal.

Se exploró nuevamente y se constató una gran masa abdominal en el flanco izquierdo. La ecografía abdominal mostró una masa abdominal retroperitoneal anterior a L1-L5 de $68 \times 39 \times 45 \mathrm{~mm}$, con puntos hiperecogénicos que sugerían calcificaciones, situada en la línea media, que dejaba libre las suprarrenales, englobaba grandes vasos y no penetraba en los forámenes vertebrales. Tras estos hallazgos, la sospecha diagnóstica fue de neuroblastoma congénito.

Durante las primeras 24 horas, se produjo una mejoría progresiva en el estado general, con normalización de la acidosis metabólica y control de la hipertensión arterial con hidralazina (dosis máxima de $1,5 \mathrm{mg} / \mathrm{kg}$ ).

Se amplió el estudio y se observó, en la bioquímica sérica, enolasa $>200 \mathrm{ng} / \mathrm{ml}$, ferritina $>2000 \mathrm{ng} / \mathrm{ml}$, lactato deshidrogenasa $>13800 \mathrm{U} / \mathrm{L}$. Catecolaminas en la orina elevadas: relación ácido homovanílico/creatinina de $333,8 \mathrm{mg} / \mathrm{g}$ (valor normal -VN- : 5,5-33), vanilmandélico/creatinina de $562,8 \mathrm{mg} / \mathrm{g}$ (VN: 4-25) y 5-hidroxiindolacético de $22,9 \mathrm{mg} / \mathrm{g}$ (VN: 1,6-6,8). La resonancia magnética abdominal mostró hallazgos compatibles con neuroblastoma retroperitoneal, sin imágenes sugerentes de afectación metastásica ganglionar ni ósea (Figura 1). El estudio de extensión descartó la afectación a distancia o la infiltración de la médula ósea.

Tras la biopsia, la anatomía patológica informó de neuroblastoma pobremente diferenciado con índice mitótico-cardiorréxico del 2-4 \% (intermedio). Histopatología favorable. Estudio

FiguRA 1. Resonancia magnética nuclear del neuroblastoma al momento del diagnóstico
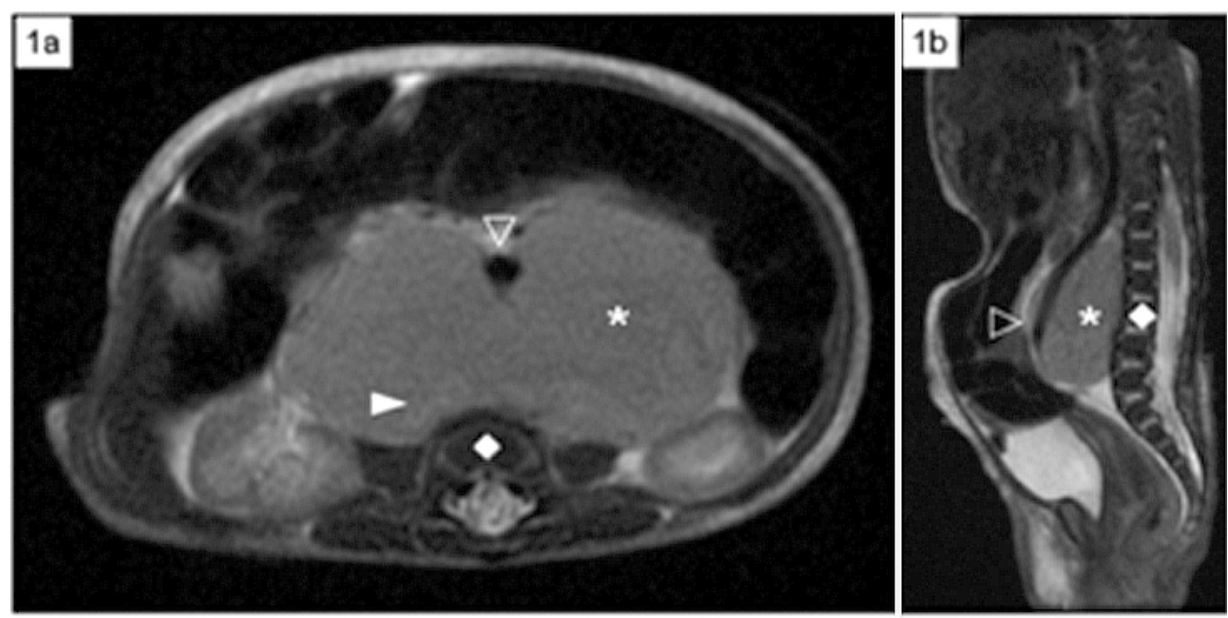

Se observa una masa sólida retroperitoneal de morfología ovalada que mide alrededor de 68 × 39 x 45 mm (asterisco). Se localiza inmediatamente anterior a los cuerpos vertebrales (rombo) de L1 a L5 sin afectación de estos, ni extensión por los forámenes. Se desplaza anteriormente a la aorta (flecha de punta hueca) y arterias ilíacas comunes, y engloba a la primera en alrededor de $270^{\circ}$ de su circunferencia. Tiene una intensidad de señal ligeramente heterogénea con pequeñas áreas hiperintensas en secuencias potenciadas en T2 sugerentes de hemorragia (flecha punta blanca).

1. a. Corte axial potenciado en secuencia T2-FSE fat-sat. 1. b. Corte coronal potenciado en secuencia T2-FSE fat-sat. 
genético: NMyc no amplificado, sin delección 1p, triploide, sin alteraciones segmentarias.

Con la alta sospecha de tumor de extirpe neuroepitelial y la presencia de signos amenazantes para la vida (hipertensión arterial, ectasia renal, plaquetas $>50000$, función hepática alterada), se decidió iniciar un tratamiento quimioterápico urgente con etopósido y carboplatino, según el protocolo europeo de tratamiento para el neuroblastoma de riesgo bajo e intermedio de la Sociedad Internacional de Oncología Pediátrica (SIOPEN, 2010). Con los resultados de la genética y de la anatomía patológica, se catalogó dentro del grupo de bajo riesgo (estadio L2). Tras el primer ciclo de quimioterapia, desaparecieron los signos amenazantes para la vida y completó un segundo ciclo al mes de vida, con una muy buena respuesta, con una reducción de la tumoración mayor del $50 \%$, pero que no permitió la cirugía. Se mantuvo una actitud expectante con observación y se manifestó la regresión completa. La hipertensión arterial se controló con hidralazina oral, que desapareció a los dos meses de vida.

Las pruebas complementarias a los 2 años y medio (26 meses tras finalizar el tratamiento) mostraron la regresión completa del tumor (sin evidencia de este en las pruebas analíticas o de imagen).

\section{DISCUSIÓN}

El diagnóstico inicial de un neonato con shock agudo es amplio; las causas más frecuentes son la sepsis de transmisión vertical y una cardiopatía congénita grave, dependiente del ductus. En ambos casos, la hipotensión es frecuente. Sin embargo, la presencia de hipertensión, junto con la masa abdominal, orientaba el diagnóstico de neuroblastoma abdominal.

El neuroblastoma congénito es un tumor embrionario derivado de células ganglionares del sistema nervioso simpático. La localización más frecuente del tumor primario es abdominal, seguida de torácica, cervical o paraespinal. La mayoría tiene síntomas debido a la masa tumoral. Lo más frecuente es la palpación de una masa abdominal, el incremento del perímetro abdominal o el dolor por la compresión de estructuras adyacentes. ${ }^{5}$

Aunque con menos frecuencia que en el feocromocitoma, puede haber una excreción aumentada de simpaticomiméticos que produzcan hipertensión arterial o incluso preeclampsia materna. ${ }^{5-7}$ En la orina, se detecta un incremento en la excreción de derivados de catecolaminas (ácido homovanílico, ácido vanilmandélico). La anatomía patológica confirma el diagnóstico. ${ }^{6}$

En nuestro paciente, la forma de presentación fue un shock desarrollado tras el nacimiento. Clínicamente, se caracterizaba como pobre perfusión periférica, acidosis metabólica grave y taquicardia con hipertensión. El diagnóstico inicial fue un shock séptico, pero la hipertensión arterial, la normalidad de los reactantes de fase aguda y la negatividad de los cultivos bacterianos indicaban un diagnóstico alternativo.

Se ha descrito la presencia de un shock como forma de presentación de un neuroblastoma por rotura de este y hemorragia o por falla cardíaca secundaria al aumento de la poscarga (secundaria a la hipertensión arterial). Otra hipótesis es la liberación de catecolaminas al torrente sanguíneo tras el nacimiento, debido a la compresión del tumor primario durante el paso por el canal del parto. ${ }^{5,8} \mathrm{El}$ incremento de los derivados de la catecolamina puede producir una elevación en las resistencias vasculares y en el consumo de oxígeno y un estado hiperdinámico con aumento de la poscarga, que, como consecuencia, produce taquicardia, hipertensión arterial, redistribución del flujo sanguíneo con disminución de la perfusión cutánea y hepática.

En nuestro paciente, los niveles de excreción de catecolaminas fueron entre 3 y 20 veces superiores a lo normal. La monitorización fetal sin incidencias, junto con la aparición de los síntomas en la primera hora de vida y su normalización posterior, sugirieron la liberación hormonal tras el parto como la causa de la clínica, como estaba descrito previamente. ${ }^{5}$ La persistencia de los síntomas, a pesar de la semivida corta de las catecolaminas, pudo ser explicada por la liberación prolongada o una alteración en su aclaramiento por la afectación hepática. ${ }^{5}$ La propia compresión de grandes vasos abdominales pudo disminuir la precarga cardíaca, lo que agravó la insuficiencia cardíaca. ${ }^{8}$ La ecocardiografía descartó la afectación miocárdica.

Esta forma de presentación es inusual y, en nuestro conocimiento, solo otro caso ha sido publicado en la literatura. ${ }^{5}$ Igualmente, fue un neonato a término con shock inicial e hipertensión, producidos por un neuroblastoma retroperitoneal. En el estudio hormonal, las catecolaminas en la orina se encontraban elevadas y se precisaron varios antihipertensivos durante su manejo. 
El tratamiento inicial, igual que con el feocromocitoma, consiste en la administración de fármacos antihipertensivos y antagonistas adrenérgicos. Durante la cirugía y el tratamiento quimioterápico, puede haber una nueva liberación de catecolaminas que requieran incrementar la terapia. ${ }^{6}$

La localización del tumor primario y la presencia de metástasis, su genética (ganancias cromosómicas completas o parciales) y la edad al momento del diagnóstico permiten estratificar a los pacientes según su probabilidad de regresión espontánea y curación. Por ello, el tratamiento oscila entre la observación y el tratamiento de soporte en los de bajo riesgo, hasta un tratamiento completo que incluya quimioterapia intensiva, cirugía, radioterapia del lecho tumoral y metástasis, trasplante de progenitores hematopoyéticos, ácido retinoico y terapia inmunológica con anticuerpos anti-GD2 e IL2. Los mayores conocimientos en la genética de estos tumores (gen $A L K, P H O X 2 B, B R C A 2$, $B R I P 1, P A L B 2^{9}$ ) y su pronóstico (amplificación del NMYC, deleción del 11p23, diploidía ${ }^{10}$ ), probablemente, ampliarán el arsenal terapéutico frente a ellos.

\section{REFERENCIAS}

1. Fernández Álvarez D, Muriel Ramos M. Tumores de la cresta neural. Pediatr Integral. 2012; 16(7):515-524.

2. Lakhoo K, Sowerbutts H. Neonatal tumours. Pediatr Surg Int. 2010; 26(12):1159-68.

3. Dhir S, Wheeler K. Neonatal neuroblastoma. Early Hum Dev. 2010; 86(10):601-5.

4. Cohn SL, Pearson AD, London WB, Monclair T, et al. The International Neuroblastoma Risk Group (INRG) Classification System: An INRG Task Force Report. J Clin Oncol. 2009; 27(2):289-97.

5. Lindner W, Behnisch W, Kunz U, Debatin KM, et al. Congenital neuroblastoma mimicking early onset sepsis. Eur J Pediatr. 2001; 160(7):436-8.

6. Seefelder C, Sparks JW, Chirnomas D, Diller L, et al. Perioperative management of a child with severe hypertension from a catecholamine secreting neuroblastoma. Pediatr Anesth. 2005; 15(7):606-10.

7. Steinmetz JC. Neonatal hypertension and cardiomegaly associated with a congenital neuroblastoma. Pediatr Pathol. 1989; 9(5):577-82.

8. Weinblatt ME, Heisel MA, Siegel SE. Hypertension in children with neurogenic tumors. Pediatrics. 1983; 71(6):947-51.

9. Orbach D, Sarnacki S, Brisse HJ, Gauthier-Villars M, et al. Neonatal cancer. Lancet Oncol. 2013; 14(13):e609-20.

10. Maris JM. Recent advances in neuroblastoma. NEnglJMed. 2010; 362(23):2202-11. 